\title{
MAC Scheduling with Low Overheads by Learning Neighborhood Contention Patterns
}

\author{
Yung Yi, Member, IEEE, Gustavo de Veciana, Senior Member, IEEE and Sanjay Shakkottai, Member, IEEE
}

\begin{abstract}
Aggregate traffic loads and topology in multi-hop wireless networks may vary slowly, permitting MAC protocols to 'learn' how to spatially coordinate and adapt contention patterns. Such an approach could reduce contention, leading to better throughput. To that end we propose a family of MAC scheduling algorithms and its general conditions, if satisfied, ensure latticethroughput-optimality (i.e., achieving any rate-point on a uniform discrete-lattice within the throughput-region). This general framework for lattice-throughput-optimality allows us to design MAC protocols which meets various objectives and conditions. In this paper, as instances of such a lattice-throughput-optimal family, we propose distributed, synchronous contention-based scheduling algorithms under graph and physical interference model that (i) is lattice-throughput-optimal, (ii) does not require node location information, and (iii) has a signaling complexity that does not depend on network size. Thus, it is amenable to simple implementation, and is robust to network dynamics such as topology and load changes. Further, we propose a heuristic, which belongs to the proposed throughput-optimal family, for achieving faster convergence, leading to a better transient throughput.
\end{abstract}

\section{INTRODUCTION}

\section{A. Motivation and Overview}

Since the seminal work [2] on throughput maximization, there has been growing interest in distributed MAC scheduling algorithms with provable throughput-guarantees over wireless multi-hop networks. The problem is to find a "throughputoptimal" algorithm, i.e., a link scheduling algorithm that stabilizes the system whenever possible, subject to the constraints on the sets of simultaneously schedulable links. Research on distributed algorithms is crucial in wireless multi-hop networks due to difficulty in having a centralized coordinator. Recent advances on this area propose various types of algorithms: maximal/greedy scheduling (e.g., [3-7]), pick-and-compare scheduling (e.g., [8,9]), and random access scheduling (e.g., $[10,11])$.

In this paper, our objective is to develop distributed, synchronous contention-based scheduling algorithms for both graph-based and physical interference model (i.e., path-loss based interference with a minimum SIR requirement for successful packet decoding at receivers) that: $(i)$ is latticethroughput-optimal, (ii) does not require node location/explicit path-loss information, and (iii) has a signaling complexity that does not depend on network size. Thus, it is amenable to simple implementation, and is robust to network dynamics such as topology and load changes. Our work is motivated by the following:

This research was supported by NSF Grants CNS-043507, CNS-0347400, CCF-0634898, and the DARPA ITMANET program. The work of G. de Veciana was supported in part by NSF Award CNS-0721532. A shorter version of this paper appeared in the Proceedings of IEEE Infocom 2007 [1]. (a) Achieving low control overhead. In a distributed implementation of link scheduling with throughput-guarantees, control overheads are unavoidable, since nodes need to exchange control messages to choose a "good" schedule over timeslots and achieve provable throughput-guarantees. Clearly, low control overhead is of great importance for both ease of implementation and higher throughput, particularly in wireless multi-hop networks with limited bandwidth resources.

(b) 'Universality' for interference models. Existing algorithms to date have mostly targeted a particular set of graph-based interference models. In a more realistic model, one would identify interference relationships based on SINR (Signal-to-Interference-Noise-Ratio), considering "aggregate" interference. There is little work on throughput-optimal algorithms under this physical interference model. The graph interference model enables us to leverage well-developed graph theoretic results. The physical interference model reflects more realism, but the complex interference coupling and aggregate interference becomes an impediment to development of a distributed scheduling algorithm with a provable throughputguarantee. Since both models (graph-based and physical interference) have their proponents, it would be useful to develop a unified scheduling framework, which allows the development of throughput-optimal distributed scheduling algorithms for both models.

In this paper, we first propose a MAC scheduling framework and associated conditions, which, if satisfied, ensure lattice-throughput-optimality, i.e., achieve any rate-point on a discrete-lattice (which can be made arbitrarily fine by a suitable choice of parameters) within the throughput-region, irrespective of underlying interference model. Next, as an instance of the proposed family of lattice-throughput-optimal algorithms, we develop a synchronized contention based algorithm, RCAMA (Randomized Contention Aware Multiple Access), which only requires very simple contention signaling on each time-slot. Further, RCAMA operates in a "dynamic" manner, i.e., schedules (determined in a distributed manner) are initially not necessarily conflict-free, but each node progressively adapts its schedule and converges to an optimal conflict-free schedule (see Figure 1). Our approach to contention signaling enables each node to 'learn' its neighborhood's contention patterns in an autonomous manner and adapt to changes in traffic load and network topology.

Note that under the physical interference model, a centralized scheduling algorithm requires exact topology knowledge (i.e., node locations, path-loss coefficients and network connectivity) to achieve throughput-optimality - this information is needed to compute the amount of interference generated by simultaneously activated nodes, which in-turn is needed for computing the optimal schedule. However, somewhat sur- 
prisingly, the proposed distributed algorithm achieves latticethroughput-optimality without centralized geographical information. To the best of our knowledge, this paper is the first to propose a distributed, throughput-optimal scheduling algorithm under the physical interference model.

We focus on a random access approach that uses memory of past (local) schedules in order to provide a higher throughputguarantee than the algorithms described in current literature (we refer to Section I-B for additional discussion). "Random access" has been popularly implemented in a practical network due to its ease of deployment. In this paper, we assume that a node has explicit knowledge of its local (long-term) offered load (i.e., offered load on each of its outgoing links). We are able to prove that this extra local-information at nodes leads to a distributed, lattice-throughput-optimal algorithm that requires only three and two stages of simple contention signaling under the physical and graph interference models, respectively, irrespective of network size.

In practice, depending on the types of services supported by the network, information on the offered load can either be explicitly given to the nodes or be measured by the nodes. If we have a guaranteed-service network based on a resource reservation signaling (e.g., RSVP [12]), the amount of load could be known a priori by nodes in the path of a reserved flow. However, in a typical best-effort service network, the amount of load is not explicitly provided to the nodes, but the nodes could measure/estimate offered load over a suitable time-period. The main motivation for RCAMA is that although individual (end-to-end) traffic loads may change quickly, the aggregates on some congested links may, in many relevant applications, change more slowly and locally. Similarly, node mobility (that leads to changes in topology and load) might be slow enough to permit a MAC scheduler to learn and exploit the offered traffic characteristics so as to quickly realize "good" schedules. Because the loads may exhibit some variation, or measurements may be noisy, a node may use an upper estimate for it.

Our scheduling framework and the proposed algorithms can be applied to both physical and graph interference model with slight modifications. However, scheduling problems are generally more challenging in the physical model due to the impact of interference generated by far-field transmissions. Thus, we focus on the physical model in this paper, and we refer the readers to our technical report [13] for graph based interference models.

\section{B. Related Work}

Scheduling achieving maximum throughput dates back to the work [2] by Tassiulas and Ephremides, where the authors show that max-weight scheduling proves to maximize throughput in constrained queueing systems. Max-weight scheduling essentially corresponds to a maximum weight independent set problem, which is known to be NP-hard, and thus requiring significant overhead. Maximal/greedy scheduling (e.g., [3$5,7]$ ) has been proposed with the objective of polynomialcomplexity overhead, however at the cost of a reduced throughput-region. The work in $[10,11]$ employs random access approach, where transmission attempt probability is decided based on the queue lengths of interfering links and just requires constant overheads with throughput-regions close to that of maximal/greedy scheduling. In this spirit (i.e., queuelength based access probability selection), related work also includes [14]. Research efforts, called a pick-and-compare approach have been developed to achieve the maximum throughput with polynomial complexity motivated by the work in [15]. The pick-and-compare based algorithms $[8,9,16]$ are essentially an "infrequent" computation of the max-weight schedule, such that complexity on each time-slot is divided into multiple time-slots $[17,18]$. Thus pick-and-compare algorithms reduce (average) complexity without loss of throughput, but incurs exponentially large delay.

Note that all related work that we have surveyed so far does not make explicit use of the offered load (i.e., statistics of the load over any link in the topology). This indicates that scheduling algorithms, which are unaware of arrival rate, pay significantly large cost in terms of control overhead or delay. As mentioned earlier, in this paper we propose an algorithm with a small knowledge of local offered load can significantly reduce control overhead as well as achieve large throughput-region. Most importantly, unlike much of the earlier work (which are predominantly for graph-based interference models), the current approach works for physical interference models as well.

We conclude this subsection with a brief survey of related work to scheduling under the physical interference model (these alorithms however have a different objective from that in this paper). The work of [19-21] develops a mathematical programming formulation for minimizing the frame size over a TDMA wireless multi-hop networks, and proposes a distributed heuristic $[19,20]$ that considers only closest interferers and a centralized heuristic which is used as a benchmark [21]. The authors in [22] define the "scheduling complexity," i.e., minimum amount of time required until every link is scheduled at least once, which is studied in an asymptotic manner. In [23, 24], the authors have focused only on computing maximum throughput under the physical interference model by jointly considering routing, MAC scheduling, and power control in an optimization framework, but no practical, throughput-optimal, distributed algorithm is presented.

\section{Main Contributions and Organization}

The main contributions of this paper are as follows:

(i) We first propose a scheduling framework (DRS: Dynamic Randomized Scheduling), that achieves any rate-point on a uniform discrete-lattice within the throughput region (i.e., lattice-throughput-optimal). To that end, we give two general conditions, which, if satisfied, ensure that an algorithm in the DRS family is lattice-throughputoptimal, and we further study their rate of convergence.

(ii) Next, as an instance of the DRS family, we propose a synchronous contention-based algorithm, RCAMA-MAX (Randomized Contention-Aware Multiple Access-MAX), where multi-stage contention signaling in conjunction with randomized time-slot selection is used. We prove lattice-throughput-optimality of RCAMA, by showing that RCAMA satisfies the two conditions in (i). The key 
ideas are summarized as a priority-based slot access with preemption and persistence properties. In spite of latticethroughput-optimality of RCAMA-MAX for general network topologies, it may not be hard to be practically be implemented due to its large power consumption in signaling. We propose a practical alternative, RCAMAVIR, whose signaling power consumption is significantly smaller than that of RCAMA-MAX, but achieves latticethroughput-optimality for many practical network topologies in wireless multi-hop networks.

(iii) We propose an adaptive variation of RCAMA, ARCAMA (Adaptive RCAMA), which again satisfies the two conditions in (i) and adaptively biases slot selection probabilities based on the past contention histories. We show via simulation that only a short duration of memory is required to increase performance, resulting in good adaptation to load/topology changes.

The rest of this paper is organized as follows: We begin with a description of the system model, notations, and definitions in Section II. Next, in Section III, we define the DRS algorithm family, and present two general conditions for a DRS algorithm to be lattice-throughput-optimal. In Sections IV and $\mathrm{V}$, we propose RCAMA as an instance of such a latticethroughput-optimal family. Finally, in Section VII, we validate our results using simulations.

\section{System Model, Notations, And Definitions}

\section{A. System Model}

We assume that time is slotted. A time-slot duration is suitably chosen to accommodate the transmission of one fixedsize packet. We model the wireless multi-hop network by a graph $\mathcal{G}(\mathcal{L}, \mathcal{V})$, where $\mathcal{L}$ and $\mathcal{V}$ denote a set of directed links and nodes, respectively. We assume that for any link between two nodes there is a counter-part in the opposite direction. We denote a directed link from node $i$ to node $j$ by $i \rightarrow j$. For concreteness, the wireless system under study has a single frequency/code and each node is time-synchronized and has a half-duplex radio.

We assume a fixed power model, where a transmitter uses the power $P$ for data transmission, and SINR (Signal-toInterference-Noise Radio) is considered to determine success or failure of a transmission.

A message from $i$ to $j$ is decodable, if

$$
\frac{G_{i j} P}{\eta_{j}+\sum_{k \in \mathcal{V}_{I}(i)} G_{k j} P} \geq \gamma
$$

where $\mathcal{V}_{I}(i)$ is the set of nodes transmitting simultaneously with $i$ on a given time-slot, $G_{i j}$ is the propagation loss from $i$ to $j$, and $\eta_{j}$ is the thermal noise power at $j$. The SINR threshold $\gamma$ depends on the desired bit rate, bit error rate, and design parameters such as modulation, coding, and so on.

In practice, in addition to interference, wireless links are prone to errors due to many other factors (e.g., fading). This leads to high packet loss rate detrimental to upper-layer performance. Thus, in many MAC protocols, reliability is provided by acknowledging transmissions and possibly retransmitting. Thus, we say that a transmission over $i \rightarrow j$ is successful, if both the data message from $i$ to $j$ and the corresponding ack message from $j$ to $i$ are decodable at $j$ and $i$, respectively, where the ack message from $j$ will be sent only when the data message is decodable at $i$.

\section{B. Lattice-Throughput-Optimality: Notation and Definitions}

Definition II.1. $A$ link schedule $\vec{A}=\left(A_{l} \in\{0,1\}: l=\right.$ $1, \ldots,|\mathcal{L}|), A_{l}$, where $A_{l}=1$ if the link $l$ is scheduled for attempted transmission, and 0 otherwise.

A link schedule $\vec{A}$ is said to be successful, if the transmissions scheduled by $\vec{A}$ are successful when they occur simultaneously. We denote the collection of all successful link schedules by $\mathcal{A}$.

Definition II.2. We define the throughput region $\Lambda$ by:

$$
\Lambda=\left\{\vec{\alpha} \mid \vec{\alpha}=\sum_{\overrightarrow{A_{i}} \in \mathcal{A}} \beta_{i} \vec{A}_{i}, \quad 0 \leq \beta_{i} \leq 1, \quad \sum_{i=1}^{|\mathcal{A}|} \beta_{i}=1\right\} .
$$

Definition II.3. For any fixed positive integer $F$, we define the $F$-lattice-throughput region $\Lambda_{F}$ by:

$\Lambda_{F}=\left\{\vec{\alpha} \mid \vec{\alpha}=\sum_{\overrightarrow{A_{i}} \in \mathcal{A}} \beta_{i} \vec{A}_{i}, \beta_{i}=\frac{k_{i}}{F}, \sum_{i=1}^{|\mathcal{A}|} k_{i}=F, k_{i} \in\{0, \ldots, F\}\right\}$.

Intuitively, $\Lambda_{F}$ is the lattice-sampling of $\Lambda$ with adjacent points having a distance of $1 / F$. Note that $\Lambda=$ $\mathrm{CL}\left(\cup_{F=1, \ldots, \infty} \Lambda_{F}\right)$, where $\operatorname{CL}(Z)$ is the closure of a set $Z$.

A scheduling algorithm $\Pi$ chooses a sequence of link schedules (which are not necessarily successful), $(\vec{A}[s]: s=$ $0,1, \ldots)$, where $\vec{A}[s]$ is the link schedule on time-slot $s$.

Definition II.4. For a fixed $F$, the offered load $\vec{\rho}$ is said to be $F$-lattice-feasible if $\vec{\rho} \in \Lambda_{F}$. A scheduling algorithm $\Pi$ is said to be $F$-lattice-throughput-optimal, if $\Pi$ stabilizes the system for any F-lattice-feasible load.

For a $F$-lattice-feasible load $\vec{\rho}$, by multiplying the offered load by $F$, we henceforth deal with positive integer-valued load, $\vec{\theta} \in \mathcal{Z}_{+}^{|\mathcal{L}|}$, i.e., $\theta_{l}$ corresponds to the number of requested time-slots over link $l$ out of $F$ time-slots. We call a group of $F$ time-slots a frame throughout this paper.

In our framework, the lattice-parameter $F$ is a systemwide parameter that is known to every node in the network a-priori. Thus, throughout this paper, we implicitly assume that the lattice-parameter, denoted by $F$, is fixed. Further, for simplicity, we use the terms "throughput-optimal" and "feasible" to refer to " $F$-lattice-throughput-optimal" and " $F$ lattice-feasible," respectively, unless explicitly needed.

\section{Dynamic RANDOMIZED SCHEDUling: CONDITIONS FOR THROUGHPUT-OPTIMALITY}

In this paper, we consider "frame-based" scheduling algorithms, where scheduling patterns are determined on a frameby-frame basis (i.e., $F$ time-slots) ${ }^{1}$, and we will see that it is sufficient to consider such class of algorithms.

Definition III.1. We define a frame schedule (FS) to be a consecutive sequence of $F$ link schedules, i.e., an $|\mathcal{L}| \times F$

\footnotetext{
${ }^{1}$ Thus, we henceforth use a term 'time-slot $s$ ' to refer to the $s$-th timeslot inside a frame. We typically use ' $\mathrm{s}$ ' and ' $\mathrm{t}$ ' to refer to the indexes of a time-slot and a frame, respectively.
} 
matrix, $C(F, \vec{\theta})=\left(c_{l s}: l=1, \ldots,|\mathcal{L}|, s=1, \ldots, F\right)$, where $c_{l s}=1$ if a transmission is scheduled over link $l$ on time slot $s$, and 0 otherwise. Further, the l-th row vector of $C(F, \vec{\theta})$, is said to be a slot schedule over l. A FS $C(F, \vec{\theta})$ is said to be feasible, if all of $F$ link schedules (column vectors) in $C(F, \vec{\theta})$ are successful.

As mentioned in Section I, we assume that a node has knowledge only of the local offered load (i.e., arrival rate) on each of its outgoing links. Thus, for all $l \in \mathcal{L}, \theta_{l}=\sum_{s=1}^{F} c_{l s}$, i.e., the number of scheduled time-slots on each link is equal to the load offered on that link.

Definition III.2. We additionally define a transmission priority, $R=\left(r_{l s}: l=1, \ldots,|\mathcal{L}|, s=1, \ldots, F\right)$ where $r_{l s}=1$ $\left(r_{l s}=0\right)$ if $c_{l s}=1$ and its priority is high (low), and NULL otherwise $\left(c_{l s}=0\right)$.

In this paper, we consider the following class of framescheduling algorithms:

Definition III.3. A dynamic randomized scheduling (DRS) algorithm randomly chooses a sequence of $(C[t], R[t]: t=$ $0,1, \ldots)$ over frames, where $C[t]$ and $R[t]$ are the $F S$ and the transmission priority at frame $t$, respectively. A randomly chosen $(C[t], R[t])$ at frame $t$ may depend on FSs of the previous, say $m$, frames. In this case we say that a DRS algorithm has history $m$. Note that in a DRS algorithm without priority, $R[t]$ is not in use.

Remark III.1. It is clear that $\vec{\theta}$ is F-lattice-feasible, if and only if there exists a feasible frame schedule $C(F, \vec{\theta})$, by Definition II.3. Our objective in this paper is to develop a DRS scheduling algorithm which finds a feasible frame schedule within a finite number of frames, and sustains the schedule thereafter, for any given feasible load. It can be easily seen that a DRS algorithm satisfying such properties achieves latticethroughput-optimality. Thus, it suffices to consider the family of DRS algorithms.

Now, we derive two conditions, which, if met, ensure that a DRS algorithm is throughput-optimal We first define a "distance" between two FSs (under the same topology and load), $C=\left(c_{l s}\right)$ and $C^{\prime}=\left(c_{l s}^{\prime}\right)$, to be:

$$
D\left(C, C^{\prime}\right)=\sum_{l=1}^{|\mathcal{L}|} \theta_{l}-\sum_{l=1}^{|\mathcal{L}|} \sum_{s=1}^{F} c_{l s} \times c_{l s}^{\prime} .
$$

Note that $D\left(C, C^{\prime}\right)=0$ implies $C=C^{\prime}$.

Definition III.4. For a given fixed load and topology, let the current frame to be $t_{i}$.

1) Finite Sustenance Condition (FSC). If $C\left[t_{i}\right]$ is feasible, $C[t]=C\left[t_{i}\right]$, w.p. $1 \forall t>t_{i}$.

2) Finite Improvement Condition (FIC). If $C\left[t_{i}\right]$ is not feasible, for any feasible FS $C^{\star}$, there is a $t<\infty$ (not dependent on $\left.C^{\star}\right)$, such that $D\left(C\left[t_{i}\right], C^{\star}\right)>D\left(C\left[t_{i}+\right.\right.$ $\left.t], C^{\star}\right)$ with positive probability.

The finite sustenance condition means that if a FS converges to a feasible one, it has to be sustained thereafter. The finite improvement condition is such that before converging to a

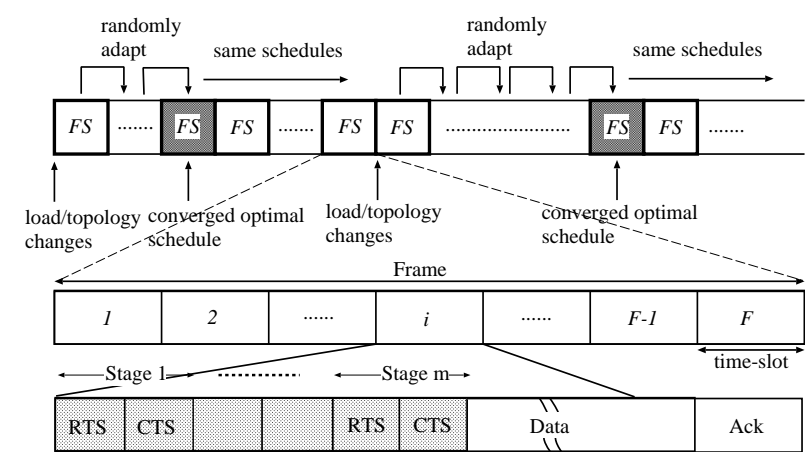

Fig. 1. Frame and slot structure of RCAMA

feasible FS, a sequence of FSs over frames tend to be progressively "closer" to a feasible FS with positive probability.

Subject to these two conditions, we have the following theorem:

Theorem III.1. For any fixed feasible offered load and topology, consider a DRS algorithm $\Pi$ which satisfies the finite improvement and sustenance conditions. We have that

1) $\Pi$ converges to a feasible FS, and thus $\Pi$ is throughputoptimal.

2) Let $\tau_{\Pi}(C)$ be the convergence time of $\Pi$ to a feasible FS for a given initial frame schedule $C$. Then, $\forall t \in \mathcal{Z}_{+}$, there exist constants $0<K_{\Pi}<\infty$ and $0<p_{\Pi}<1$, such that $\operatorname{Pr}\left\{\tau_{\Pi}(C)>t K_{\Pi}\right\} \leq p_{\Pi}^{t}$.

The sketch of proof is as follows: First, it is easily seen that a sequence of FSs over frames forms a Markov chain. Then, the finite improvement condition implies that we can construct a converging path to a feasible FS (say, $C^{\star}$ ) within a finite time, since $D\left(C, C^{\star}\right)$ is upper-bounded. The complete proof is presented in the Appendix.

The finite sustenance and improvement conditions described above enable us to verify throughput-optimality of an instance of the DRS family. In addition, it allows customization or enhancement of an algorithm with its throughput-optimality maintained, as long as the modified version satisfies those conditions. In this paper, we develop a "base-line" DRS algorithm with history 1 . We later discuss how such baseline algorithms with history 1 can be extended to adaptive versions with multiple frame histories for better adaptation to load/topology changes and faster convergence to the optimal schedule in Section VI.

\section{RCAMA: OVERVIEW AND PER-FRAME OPERATION}

\section{A. Overview}

The general frame and time-slot structure of RCAMA are shown in Figure 1. A time-slot is divided into two parts: time for contention signaling and time for data and ack transmission $(\mathrm{TX})^{2}$. We will describe RCAMA by dividing its behavior into two different time-scales: ( $i$ ) per-frame operation, where each node randomly determines the slot-schedules for the TXs over its adjacent outgoing links, and (ii) per-slot operation, where a node initiates a RTS/CTS-like contention signaling

\footnotetext{
${ }^{2}$ For notational simplicity, we use the term ' $\mathrm{TX}$ ' to refer to the word 'transmission' throughout this paper.
} 
to resolve contentions and implicitly learn contention patterns in the neighborhood. In this section, we describe only perframe operation, and per-slot operations will be discussed in Sections V.

The RCAMA is designed to ensure the following two properties:

1) Persistence: A successful TX at a given time-slot at the current frame persists on the same slot at the next frame.

2) Preemption: An unsuccessful TX can preempt a timeslot (with positive probability) used by a persistent successful TX.

As discussed earlier, it suffices to show that the system converges to a feasible FS to achieve throughput-optimality. By the persistence property, once the system reaches a "good" (i.e., feasible) FS, it stays in that FS. Preemption property ensures that there is no deterministic "winner-loser" relationships among TXs, and enables the system to avoid deadlocks, i.e., being stuck in a "bad" FS. These two properties ensure that the system will visit arbitrary FSs, and finally reach a feasible FS, which is sustained thereafter. We satisfy these two properties by assigning priority to scheduled TXs. More specifically, by assigning high priority to unsuccessful TXs and low priority to persistent successful TXs, respectively, we allow a newly scheduled unsuccessful TX on a time-slot to beat existing successful ones.

In addition to provable throughput-optimality, by using a low-cost contention signaling (i.e., message complexity does not depend on network size), the algorithm can adapt to load and topology changes by "learning" local contention patterns. In other words, RCAMA does not need any explicit mechanism to inform the nodes of such network changes, and it automatically avoids the situation where multiple timeslots are commonly accessed by interfering links. Further, application of non-uniform time-slot access probability for unsuccessful TXs enable the system to learn local contention levels, and to distribute scheduled TXs at different time-slots in a more efficient manner (see Section VI).

We note that a similar idea of using multiple priorities was introduced in Z-MAC [25]. However, Z-MAC considers only the graph-based interference model, and its major objective of multiple priorities is to solve the hidden terminal problem with no provable throughput-guarantee, whereas we use twolevel priority to get both provable convergence and throughputguarantee.

\section{B. Per-Frame Operation: Randomized Slot-Selection}

When each frame starts, each node (say, $v \in \mathcal{V}$ ) determines the slot-schedules and contention priorities for the TXs over its adjacent outgoing links. To do this, the following simple rules are used:

Rule IV.1 (Slot and Priority Selection Rule).

(i) A successful TX on time-slot $s$ at frame $t-1$ persists on the same time-slot $s$ at frame $t$, with priority set to be low.

(ii) If a TX was unsuccessful at frame $t-1$, a time-slot is randomly selected from the time-slots not already taken in (i), and its priority is set to be high.

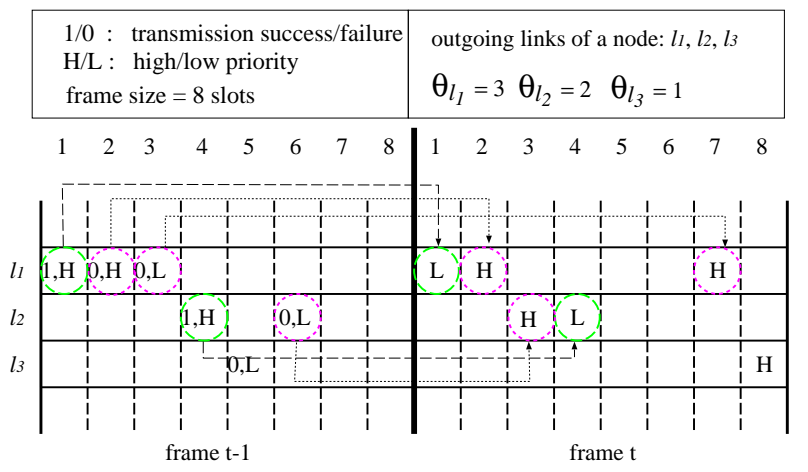

Fig. 2. Example of Rule IV.1: Since at frame $t-1$, the TX over $l_{1}$ on timeslot ' 1 ' and over $l_{2}$ on time-slot ' 4 ' were successful, these TXs are scheduled once again with low contention priority at the same time-slot positions at frame $t$. For the unsuccessful TXs over $l_{1}$ on time-slots ' 2 ' and ' 3 ', we randomly choose two time-slots of the remaining time-slots, which were not taken by previously successful TXs (i.e., the node does not consider time-slots ' 1 ' and ' 4 ' in this random selection). In the example, time-slot ' 2 ' and ' 7 ' are selected, and they are scheduled with high contention priority.

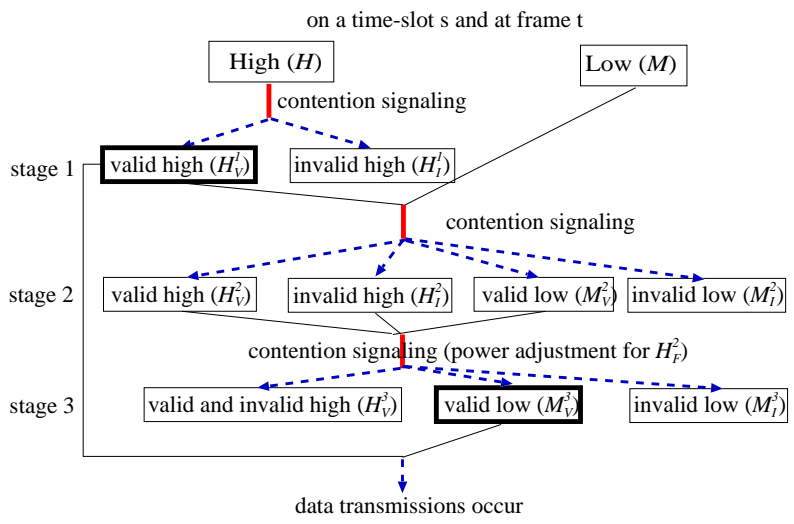

Fig. 3. Three-stage contention signaling in RCAMA under the physical interference model

Rule IV.1(i) corresponds to the persistence property. Preemption property is satisfied by Rule IV.1(ii) in conjunction with the proposed multi-stage signaling in Section V. An example of Rule IV.1 is given in Figure 2.

\section{Per-Slot Operation: Physical Interference MODEL}

\section{A. Basic Algorithm}

Following the slot-schedules as explained in Section IV-B, on each time-slot, nodes use the three-stage (synchronized) RTS/CTS contention signaling mechanism to resolve contentions, and data/ack TXs follow (see Figure 3 for a representative, pictorial algorithm description).

Definition V.1. A scheduled TX over $i \rightarrow j$ is said to be valid, if $j$ decodes the RTS from $i$, and $i$ decodes the CTS from $j$.

Note that in our three-stage signaling, the validity of a TX does not imply success of the TX, i.e., even if RTS/CTS are decoded, the data TX or ack reception can fail. For this reason, we differentiate between validity and success of a TX.

We denote by $\mathcal{H}$ (resp. $\mathcal{M}$ ) a set of links scheduled (on a given time-slot and frame) with high (resp. low) priority, $\mathcal{H}, \mathcal{M} \subset \mathcal{L}$. At each stage, contention signaling is conducted 
for high and/or low priority TXs. We use the notations $\mathcal{H}_{V}^{i}$ and $\mathcal{H}_{I}^{i}$ to refer to valid and invalid high priority TXs at stage $i$, respectively. Similarly, $\mathcal{M}_{V}^{i}$ and $\mathcal{M}_{I}^{i}$ are used for low priority TXs.

(i) Stage 1: Contention signaling is performed for only the TXs in $\mathcal{H}$, based on which $\mathcal{H}_{V}^{1}$ and $\mathcal{H}_{I}^{1}$ are determined (note that $\mathcal{H}_{V}^{1} \cup \mathcal{H}_{I}^{1}=\mathcal{H}$ ). The three-stage contention signaling is constructed to ensure that data TXs occur over the links in $\mathcal{H}_{V}^{1}$, irrespective of the results of the subsequent stages 2 and 3. However, their success is not guaranteed, because TXs in $\mathcal{H}_{V}^{1}$ could fail if their actual data/ack TXs occur together with TXs in $\mathcal{M}$.

We will later show that it suffices to guarantee the success of all TXs in $\mathcal{H}_{V}^{1}$ on each time-slot for throughput-optimality (see Theorem V.1). Thus, the objective of subsequent stages 2 and 3 is to ensure the success of TXs in $\mathcal{H}_{V}^{1}$.

(ii) Stage 2: Contention signaling is performed for the TXs in $\mathcal{H}_{V}^{1}$ and the TXs in $\mathcal{M}$, based on which $\mathcal{H}_{V}^{2}, \mathcal{H}_{I}^{2}$ $\overline{\mathcal{M}_{V}^{2}}$, and $\mathcal{M}_{I}^{2}$ are determined. Note that $\mathcal{H}_{V}^{2} \cup \mathcal{H}_{I}^{2}=\mathcal{H}_{V}^{1}$, and $\mathcal{M}_{V}^{2} \cup \mathcal{M}_{I}^{2}=\mathcal{M}$. The role of this stage is to identify high priority TXs in $\mathcal{H}_{V}^{1}$, which fail due to interference from low priority TXs, i.e., identify $\mathcal{H}_{I}^{2}$.

(iii) Stage 3: Contention signaling is performed again for the TXs in $\mathcal{H}_{V}^{1}$ and only for the TXs in $\mathcal{M}_{V}^{2}$. Recall that our preemption property for throughput-optimality is intended to ensure the success of high priority TXs in $\mathcal{H}_{V}^{1}$. The objective of Stage 3 is to invalidate low priority TXs, which can cause the TXs in $\mathcal{H}_{I}^{2}$ to fail (note that TXs in $\mathcal{H}_{V}^{2}$ will be successful even with interference by low priority TXs). To that end, we employ signaling power adjustment in RTS/CTS signaling for TXs of $\mathcal{H}_{I}^{2}$, i.e., the transmitters and the receivers in $\mathcal{H}_{I}^{2}$ adjust their signaling powers appropriately, such that interfering low priority TXs are invalidated.

(iv) Data/ack TXs: Data TXs occur for TXs in $\mathcal{H}_{V}^{1}$ and TXs in $\mathcal{M}_{V}^{3}$. ACK messages are sent back to the transmitters by the receivers which can decode data.

An example of the three-stage contention signaling in RCAMA is exemplified in Figure 4. It shows how it operates and converges to a feasible FS.

We note that transmission power control for signaling, which is similar to signaling power adjustment in this paper, has been proposed with the main objective of throughput improvement (see [26] and references therein). The approaches in [26], however, do not consider the physical interference model and they do not provide a study of provable performance guarantees (i.e., no throughput-optimal properties).

\section{B. Signaling Power Adjustment and Throughput-Optimality}

The remaining question is how to compute the adjusted powers for TXs in $\mathcal{H}_{I}^{2}$ in an efficient, distributed manner, which we will discuss in this section.

We will use the notation $\mathcal{H}_{I}^{2}(s)[t]$ to explicitly refer to $\mathcal{H}_{I}^{2}$ on the time-slot $s$ at frame $t$. We first let $\vec{P}_{s}^{A}[t]=\left(\overrightarrow{P^{r}}, \overrightarrow{P^{c}}\right)_{s}[t]$, $\overrightarrow{P^{r}}=\left(P_{l}^{r}\right), \overrightarrow{P^{c}}=\left(P_{l}^{c}\right), l \in \mathcal{H}_{I}^{2}(s)[t]$ be the adjusted signaling power vector on time-slot $s$ and frame $t$ at stage 3 , where $\overrightarrow{P^{r}}$ and $\overrightarrow{P^{c}}$ corresponds to the powers for sending RTS and CTS messages, respectively.

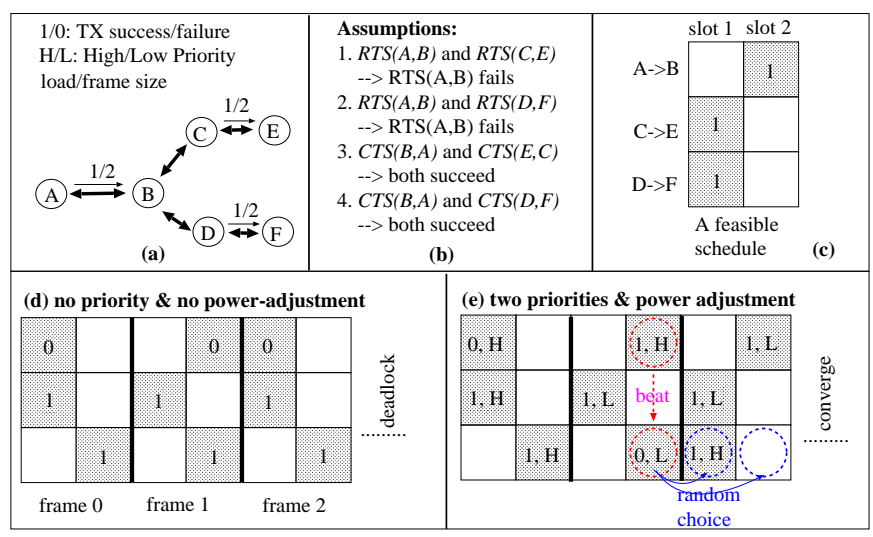

Fig. 4. Example of RCAMA: In absence of contention priority and signaling power adjustment, TX over $A \rightarrow B$ keeps failing with either choice of timeslot '1' or '2', since RTS from $A$ is not decodable at $B$ due to interference from either $C$ or $D$, over frames. However, in RCAMA, from Rule IV.1, the unsuccessful TX over $A \rightarrow B$ at frame ' 0 ' is assigned high priority at frame ' 1 ,' and due to stages 2 and 3, $B$ adjusts the power for its CTS (destined to A and broadcast to $D$ ), such that CTS from $F$ is not decodable at $D$ (see the frame 1 in (b)). The same procedure can be applied when TXs over $A \rightarrow B$ and $C \rightarrow E$ are assigned high and low on a same time-slot, respectively. By this procedure, the system ultimately converges to a feasible FS.

Definition V.2. For any fixed topology and load, consider a sequence of adjusted signaling power vectors, $\left(\vec{P}_{s}^{A}[t]\right.$ : $s=1, \ldots, F, t=0,1, \ldots$,$) . RCAMA is said to satisfy High$ Priority Condition (HPC) with $\left(\vec{P}_{s}^{A}[t]\right)$, if with $\vec{P}_{s}^{A}[t]$, all the TXs in $\mathcal{H}_{V}^{1}(s)[t]$ are successful, over any time-slot and frame.

As described in Section IV-A, Definition V.2 corresponds to a condition ensuring that "good" high priority TXs (i.e., valid TXs at stage 1) are successful. Now, Theorem V.1 implies that it suffices to guarantee the success of TXs in $\mathcal{H}_{V}^{1}$ by using sufficiently large adjusted power in stage 3 for throughputoptimality of RCAMA. Recall that $\mathcal{H}_{V}^{1}=\mathcal{H}_{V}^{2} \cup \mathcal{H}_{I}^{2}$, and TXs in $\mathcal{H}_{V}^{2}$ are guaranteed to be successful even with interference by low priority TXs.

Theorem V.1. For any given fixed topology and load, suppose that RCAMA satisfies HPC with $\left(\vec{P}_{s}^{A}[t]\right)$, then

1) RCAMA satisfies the finite sustenance and improvement conditions. Thus, by Theorem III.1, it is throughputoptimal.

2) RCAMA satisfies HPC with any $\left(\vec{Q}_{s}^{A}[t]\right)$, where $\vec{Q}_{s}^{A}[t] \geq$ $\vec{P}_{s}^{A}[t], s=1, \ldots, F, t=0,1, \ldots$, in element-wise.

This result enables us to develop the following simple, distributed throughput-optimal algorithm:

RCAMA-MAX: All the signaling power adjustment (i.e., $\left.\vec{P}_{s}^{A}[t]\right)$ are set to be $P_{\max }$, where $P_{\max }$ is the amount of signaling power, such that signaling with $P_{\max }$ in a TX invalidates all other simultaneously scheduled TXs.

The assumption that $P_{\max }$ exists is reasonable for wireless multi-hop networks deployed of a finite size. The following immediate corollary follows:

Corollary V.1 (RCAMA-MAX). For any fixed topology and feasible load, RCAMA-MAX satisfies HPC, and thus is throughput-optimal.

Remark V.1. Note that under the physical interference model, 


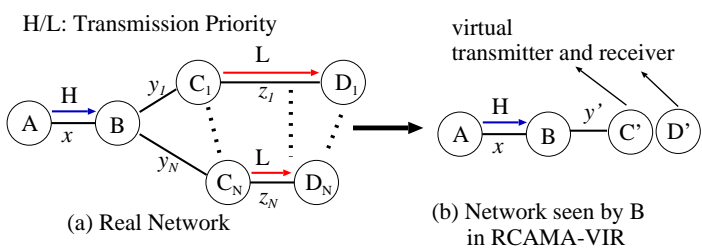

Fig. 5. Example of RCAMA-VIR: we have one high priority and $N$ low priority TXs scheduled on a same time-slot. The high priority TX is clearly valid at stage 1 . At stage 2 , suppose that at stage 2 an RTS over $A \rightarrow B$ is not decodable due to the aggregate interference of RTSs from $C_{i}$ to $D_{i}$, $i=1, \ldots, N$. Now, $B$ assumes that its RTS decoding failure is due to a single virtual low priority TX. By estimating such aggregate interference, $B$ computes the distance from itself to $C^{\prime}$ (the virtual transmitter). In the CTSslot of stage 3 , B sets the sufficiently large CTS power to invalidate a CTS from $D^{\prime}$ (the virtual receiver of $C^{\prime}$ ), based on the "worst-case" assumption that there does not exist a signal power path-loss between $C^{\prime}$ and $D^{\prime}$.

a centralized algorithm needs information on node locations and network connectivity to achieve throughput-optimality. Surprisingly, however, Corollary V.1 implies that there exists a distributed throughput-optimal scheduling algorithm that does not need such centralized topology information.

In spite of the provable throughput-optimality and the fully distributed nature of RCAMA-MAX, it may not be a practical algorithm, since for a large-scale multi-hop network, $P_{\max }$ should be very large. This is not a desirable feature leading to low efficiency of energy utilization and poor transient throughput. In other words, with RCAMA-MAX, every low priority TXs will fail, and only high priority TXs surviving stage 1 will succeed. The main observation behind this limitation of RCAMA-MAX is that we need to consider the "worst-case," i.e., the case when a large number of far field low priority TXs interfere with a high priority TX (which was valid at stage 1). However, it is known that interference is dominated by a small number of nearby transmissions mainly due to nonlinear signal power loss. Using this observation, in the next section, we propose a new distributed algorithm, RCAMAVIR, which uses far lower powers than $P_{\max }$, but still guarantees throughput-optimality under reasonable assumptions.

\section{RCAMA-VIR}

The main idea in RCAMA-VIR is to use a sufficiently high power (but not as large as $P_{\max }$ in Stage 3 signaling), such that low priority interferers of $\mathcal{H}_{I}^{2}$ can be suppressed. This is done by estimating (and developing bounds) on the interference power.

In this section, we assume the following: (i) A receiver can only measure the total received signal power (the desired signal power plus interference) and know a boolean result about the target SINR (i.e., the target SINR is larger than the threshold $\gamma$ or not $)^{3}$; (ii) the propagation loss is modeled by $G_{i j}=1 / d(i, j)^{\alpha(i, j)}$, where $d(i, j)$ is the distance between nodes $i$ and $j$, and $\alpha(i, j)$ is an "effective" path loss exponent (which may depend on the node-pair), for which each node knows (lower and upper) bounds (i.e., $\underline{\alpha} \leq \alpha(i, j) \leq \bar{\alpha}$ ); (iii)

\footnotetext{
${ }^{3}$ Note that we do not assume that the receiver is able to know the exact SINR value as well as individual or even aggregate pure interference generated by other transmissions.
}

the system is interference-limited ${ }^{4}$.

The transmitter $s(l)$ and the receiver $d(l)$ of link $l \in \mathcal{H}_{I}^{2}$ perform the following procedures:

\section{RCAMA-VIR:}

1) $d(l)(s(l))$ estimates the aggregate interference generated by low priority TXs during RTS (CTS) slot, and assumes that such interference is caused by the transmitter (receiver) of a single virtual low priority TX. (see Section V-D for discussion on estimation of the aggregate interference).

2) $d(l)(s(l))$ computes an upper-bound on the distance to the transmitter (the receiver) of the virtual TX. This upper-bound is computed based on the bounds on the path loss exponent (i.e., $\underline{\alpha} \leq \alpha \leq \bar{\alpha}$ ), and the interference estimation in (i).

3) By assuming that there is no power path-loss between the virtual transmitter and receiver, $d(l)(s(l))$ computes the adjusted CTS (RTS) power, required to invalidate the virtual TX.

An example of RCAMA-VIR is shown in Figure 5. Note that RCAMA-VIR may not be throughput-optimal, when many far field low priority transmissions are interfering a high priority transmission. However, we will show that RCAMA-VIR achieves throughput-optimality under reasonable assumptions (see Theorem V.2).

\section{Estimation of Interference}

Note that the major difference between Stages 1 and 2 is the existence of low priority TXs. Thus, it is intuitive to use measurement of the total received signal powers at Stages 1 and 2 and using their differences to estimate the interference by low priority TXs.

Consider a TX $l \in \mathcal{H}_{I}^{2}$. We denote by $\hat{R}_{d(l)}^{1}\left(\right.$ resp. $\left.\hat{C}_{s(l)}^{1}\right)$, the total received signal power on RTS (resp. CTS) slots at Stage 1 by $d(l)$ (resp. $s(l)$ ). Similarly, we use the notations $\hat{R}_{d(l)}^{2}$ and $\hat{C}_{s(l)}^{2}$, at Stage 2. We also let $I_{d(l)}^{r}$ and $I_{s(l)}^{c}$ be the exact aggregate low priority interference to $d(l)$ and $s(l)$. To estimate the interference by low priority transmitters and receivers, we use the values defined in the following: $\hat{I}_{d(l)}^{r} \triangleq$ $\hat{R}_{d(l)}^{2}-\hat{R}_{d(l)}^{1}$ and similarly, $\hat{I}_{s(l)}^{c} \triangleq \hat{C}_{s(l)}^{2}-\hat{C}_{s(l)}^{1}$.

Using the above method for estimation, we have

$$
\hat{I}_{d(l)}^{r} \leq I_{d(l)}^{r}, \quad \hat{I}_{s(l)}^{c} \leq I_{s(l)}^{c},
$$

since we have

$$
\begin{aligned}
\hat{I}_{d(l)}^{r} & =\hat{R}_{l}^{2}-\hat{R}_{l}^{1} \\
& =\hat{R}_{l}^{2}\left(\mathcal{H}_{V}^{1}\right)+\hat{R}_{l}^{2}(\mathcal{M})-\left(\hat{R}_{l}^{2}\left(\mathcal{H}_{V}^{1}\right)+\hat{R}_{l}^{2}\left(\mathcal{H}_{I}^{1}\right)\right) \\
& =\hat{R}_{l}^{2}(\mathcal{M})-\hat{R}_{l}^{2}\left(\mathcal{H}_{I}^{1}\right) \leq I_{d(l)}^{r}\left(\because R_{l}^{2}(\mathcal{M})=I_{d(l)}^{r}\right),
\end{aligned}
$$

where $\hat{R}_{l}^{i}(\mathcal{A})$ corresponds to the total received power by a receiver of link $l$ from RTS TXs in $\mathcal{A}$ during stage $i$. Similarly, we also have that $\hat{I}_{s(l)}^{c} \leq I_{s(l)}^{c}$.

\footnotetext{
${ }^{4}$ In this system, the link operates at a sufficiently high $\gamma$ (SINR threshold), so that the effect of thermal noise is negligible as compared to the interference. However, this can be readily extended to the more general assumption that $0 \leq \eta_{j} \leq \epsilon \times$ (interference), where $\epsilon$ is the ratio of thermal noise to the total interference.
} 
In other words, our estimation is a lower-bound on the exact interference by low priority TXs. This lower-bound in the interference estimation and the bounds on the path loss exponent lead to an upper-bound on the distance to the transmitter/receiver of the virtual TX, which is used in the proof of throughput-optimality of RCAMA-VIR.

Theorem V.2 (RCAMA-VIR). Suppose that there exists a maximum distance of interference between nodes and a maximum number of interferers, denoted by $d_{\text {int }}$ and $N_{\text {int }}$, respectively. If $\sqrt[2 \alpha]{N_{\text {int }}}\left(d_{\text {int }}\right)^{\bar{\alpha} /(2 \underline{\alpha})} \leq d_{\text {min }}$, where $d_{\text {min }}$ is the minimum distance between two nodes, then RCAMA-VIR satisfies HPC. Thus, it is throughput-optimal from Theorem V.1.

Theorem V.2 implies that if the inter-node distance is sufficiently large, i.e., node density in a plane is not too high and nodes are distributed in a sufficiently uniform manner, throughput-optimality is provably guaranteed in RCAMAVIR. The proof is presented in Appendix.

Numerical Example V.1. As a numerical example, consider the case when $d_{i n t}=2 \times d_{\text {min }}$ (a typical setting in the IEEE 802.11 DCF by assuming that transmission rage is set to be $\left.d_{\text {min }}\right)$ for different values of bounds on path-loss exponents and $N_{\text {int }}$, given by:

$$
\begin{aligned}
& d_{\text {min }} \geq 2.5 m \text { if } \bar{\alpha}=\underline{\alpha}=3, N_{\text {int }}=2, \\
& d_{\text {min }} \geq 4 \mathrm{~m} \quad \text { if } \bar{\alpha}=\underline{\alpha}=4, N_{\text {int }}=16, \\
& d_{\text {min }} \geq 8 \mathrm{~m} \quad \text { if } \bar{\alpha}=4, \underline{\alpha}=3, N_{\text {int }}=4 .
\end{aligned}
$$

As discussed earlier, due to non-linear path-loss exponents, the number of interferers affecting other simultaneously scheduled TXs seems to be quite limited, i.e., $N_{\text {int }}$ is small, where we have more relaxed condition on $d_{m i n}$, which still gives a provable guarantee on performance.

\section{ARCAMA (ADAPTIVE RCAMA)}

Note that RCAMA chooses new time-slots for unsuccessful TXs with equal probability in the subsequent frames. In fact, one can potentially increase the rate of convergence or adapt to load changes more effectively by intelligently guessing which time-slot is likely to be successful and by biasing the time-slot access probability. As an example, a time-slot with consecutive success is highly likely to be "safe", so that it would be beneficial to sustain the corresponding time-slot with higher probability at the next frame than other time-slots. In this section, we propose a general family of variations of DCAMA, ARCAMA (Adaptive RCAMA) family (a subset of the DRS family), which adaptively assigns different time-slot access probabilities, depending on the past contention history. This provides ARCAMA with a more efficient learning of local contention patterns, leading to more robustness to network changes. As shown in Proposition VI.1 below, such variations of RCAMA inherit all throughput-optimal properties.

To that end, each link is assigned its own slot weight vector, and the individual nodes maintain slot weight vectors for its adjacent outgoing links. This slot weight vector is updated every frame, mainly based on the TX results (success or failure) at the past frames. To increase/decrease the slot weight vector, we define the time-slot status, which corresponds to the result of past TXs on the corresponding time-slots. Then, the
TABLE I

WEIGHT INCREASE/DECREASE, $S_{s}^{l}[t]$ : SLOT STATUS OVER LINK $l$ ON SLOT $s$ AT FRAME $t$

\begin{tabular}{|c|c|c|c|}
\hline$S_{s}^{l}[t-3]$ & $S_{s}^{l}[t-2]$ & $S_{s}^{l}[t-1]$ & Weight Inc/Dec \\
\hline \hline SUCC & SUCC & SUCC & $-D_{1}$ \\
\hline FAIL/IDLE & SUCC & SUCC & $-D_{2}$ \\
\hline FAIL & FAIL & FAIL & $+I_{1}$ \\
\hline SUCC/IDLE & FAIL & FAIL & $+I_{2}$ \\
\hline
\end{tabular}

slot access probability is set to be inversely proportional to the current weight. This biased probability is used for selecting time-slots for unsuccessful TXs. Also, by setting a minimum and maximum for each weight, we can avoid pathological cases (e.g., the time-slot access probability could be arbitrarily small or close to ' 1 '), i.e., there exist $\bar{w}$ and $\underline{w}$, such that $1 \leq \underline{w}<\bar{w}<\infty$ and $\forall s \in\{1,2, \ldots, F\}, \forall l \in \mathcal{L}$, and $\forall t>0$, $\underline{w} \leq w_{s}^{l}[t] \leq \bar{w}$, where we denote the slot weight vector of link $l$ at frame $t$ by $\vec{w}^{l}[t]=\left(w_{s}^{l}[t]: s=1 . \cdots, F,\right)$.

Proposition VI.1. For any fixed topology and feasible load and any positive integer $m<\infty$, in ARCAMA with history $m$, Theorems V.1 and V.2. still hold.

We skip the proof for brevity, since it is analogous to those for RCAMA.

\section{Simulations}

In this section, we evaluate the performance of the RCAMA and ARCAMA by comparing them to the base-line RANDOM algorithm. The RANDOM algorithm determines slotschedules (based on the requested loads) in a purely random manner at each frame, and uses a single-level RTS/CTS signaling to gain access to the channel. We choose the RANDOM algorithm as a base-line, since it is similar to Aloha-like strategy (a "standard" algorithm for link scheduling), and behaves like a slotted version of a CSMA-like contentionbased scheme.

Weight maintenance algorithm. We use a simple weight maintenance algorithm based on three frame contention history in ARCAMA, where we increase (decrease) a weight more aggressively for back-to-back failures (successes) on a slot over the past three frames. We expect to see even better performance increase when more sophisticated maintenance algorithms are used. The intuition for these choices is that more back-to-back successes at a time slot indicate that the offered loads around the corresponding node at that time-slot are relatively low (i.e., less "congested"), and transmissions in that time-slot are likely to be successful in the future. Similar intuition is applied for back-to-back failures. We have three kinds of time-slot status: SUCC (FAIL), where a transmission occurs and are successful (unsuccessful), and IDLE otherwise. Table I shows the (additive) increase/decrease parameters to adapt slot weights based on the past three transmission result histories, respectively. The parameters are chosen such that $D_{1}>D_{2}>0$, and $I_{2}>I_{1}>0$. We have used $D_{1}=I_{1}=3$, $D_{2}=I_{2}=1$, in all simulation results, where the maximum and minimum weights (i.e., $\bar{w}$ and $\underline{w}$ ) are set to 30 and 1 .

\section{A. Physical Interference Model}

Simulation environment. We simulate wireless multi-hop networks with nodes which are randomly distributed in a 
$1000 \times 1000$ meter-square area. Thermal noise power at each receiver (i.e., $\eta_{j}$ ), the minimum required SINR level (i.e., $\gamma$ ), and the transmit power level (i.e., $P$ ) are set to be $-90 \mathrm{dBm}, 18$ $\mathrm{dB}$, and $15 \mathrm{dBm}$, respectively. The frame size is 10 time-slots.

Different signaling power adjustment schemes. First, we investigate the effect of different signaling power adjustment schemes on the throughput performance and energy consumption at the "steady" state (i.e., no load or topology changes for some time).

Figure 6(a) shows the network topology and link connectivity generated at random using the parameters above. Figure 6(b) shows a trace of the used powers for different RCAMA versions. Figure 6(c) shows the performance of RCAMA and ARCAMA algorithms for a normalized load by a randomly chosen maximally feasible load ${ }^{5}$, which varies from $50 \%$ to $100 \%$. We measure the aggregate normalized throughput for every varying load over 3000 frames. Each point in the graph is the mean value of 50 simulation experiments with different random seed values. In the simulation results, (A)RCAMA-NOR represents the (A)RCAMA without signaling power adjustment at stage 3 . Similarly, Figure 6(d) shows the aggregate average power used in contention signaling per one successful transmission for different values of normalized load. With both simulation results, we observe that the algorithms without power adjustment.

From these simulation results, we observe the following: (i) ARCAMA has better transient throughput than RCAMA, (ii) With both ARCAMA and RCAMA, the algorithm without power adjustment has greater transient throughput than other throughput-optimal versions with power adjustment (i.e., (A)RCAMA-VIR and (A)RCAMA-MAX), as well as better energy saving.

Note that, in practice, we may need lower powers than those used by RCAMA-VIR, and the condition on $d_{\min }$ in Theorem V.2 can be relaxed. This is because RCAMA-VIR is conservatively designed again by considering the point-ofview from one single high priority $\mathrm{TX}$ and other low priority TXs for the probable throughput-optimality. In other words, we have not considered the fact that other high priority TXs, which were valid at stage 1 , also generate interference to interfering low priority TXs, and interference among lowpriority TXs still exists. In fact, as seen above from the simulation results, RCAMA with no signaling power adjustment has a better (transient) performance than RCAMA-MAX and RCAMA-VIR even if it is not provably throughput-optimal. Essentially, overall higher performance than RANDOM is due to accessing the channel with two-level priority, which significantly reduces contentions.

Adaptation to load changes. In this simulation, we investigate the effect of network changes in load on the performance of RCAMA-NOR and ARCAMA-NOR algorithm, again for the network topology in Figure 6(a). We generate time-varying loads by a random walk model, where we first determine a normalized offered load of $60 \%$ by a randomly chosen maximally feasible load. Then, at the beginning of each frame we randomly choose $L_{c h}$ links and increase their link loads

\footnotetext{
${ }^{5} \mathrm{~A}$ load is said to be maximally feasible if the resulting system load becomes infeasible with any load increase anywhere in the network.
}

by one slot with probability $P_{I}$, decrease their link loads with probability $P_{D}$, or stay at the current load (i.e., no change) with probability $1-P_{I}-P_{D}$. For simplicity, in the simulation, we set $\hat{P} \triangleq P_{I}=P_{D}$. Thus, higher values of $\hat{P}$ corresponds to a faster load change with time. Then, the mean load change time (MLCT) over $L_{c h}$ links is $1 /(2 \hat{P})$ frames.

Figure 7(a) shows an example trace of throughput (i.e., number of successful transmission slots) for $\mathrm{MLCT}=25$ frames and $L_{c h}=5$, where we observe that ARCAMA algorithm tracks the actual load very well, resulting in nice adaptation to time-varying load changes. Figure 7(b) shows that the throughput (over 50000 frames) normalized by the actual (time-varying) offered load for different values of MLCTs $\left(L_{c h}=1\right)$ varying from 25 to 100 frames, where the error bars represent the maximum and minimum values of 10 simulations with different random seed values (i.e., different load changing patterns). For a network with a link capacity of $10 \mathrm{Mbps}$, and a frame-size of 10 (which corresponds to a $10 \mathrm{msec}$ frame duration), this corresponds to a load change ranging from once every $250 \mathrm{msec}$ to once every 1 seconds. We observe that with ARCAMA algorithm, the normalized throughput is above $90 \%$, whereas the RANDOM achieves about $60 \%$.

\section{APPENDIX}

We first observe that Rule IV.1 satisfies the Property VII.1. It basically says that any TX scheduled at some slot $s$ could be re-scheduled with positive probability. In particular, for a successful TX, probability that the same time-slot is chosen is ' 1 .' We will use this property in the proof of throughputoptimality of RCAMA algorithm in Section V-B.

Property VII.1. For any time-slot $s$, and link $l$, there exists a positive probability that $c_{l s}[t-1]=c_{l s}[t]$, irrespective of $c_{l^{\prime} s^{\prime}}[t-1], l^{\prime} \neq l, s^{\prime} \neq s$.

\section{B. Proof of Theorem III.1}

Proof of Theorem III.1(i): From Remark III.1, for the proof of throughput-optimality, we will show that the system "converges" to a feasible frame schedule. For a DRS algorithm with history $m$, it is easily seen that a sequence of $(C[t], R[t])$ over frames forms a Markov chain, by defining a system state as

$\mathbf{X}_{m}[t] \triangleq((C[t-m+1], R[t-m+1]), \cdots,(C[t], R[t]))$,

and we let $C[n]=R[n]=0$, when $n<0$, for the initial conditions.

Then, from the standard Markov chain theory and the finite sustenance condition, it suffices to show that from any state $\mathbf{X}_{m}[0]$, where $C[0]$ is infeasible, we can construct a finite sequence of times $0=t_{i}<t_{2}<\ldots<t_{n}<\infty$, such that $C\left[t_{n}\right]$ in $\mathbf{X}_{m}\left[t_{n}\right]$ is feasible.

Suppose that $C\left[t_{i}\right]$ in $\mathbf{X}_{m}\left[t_{i}\right]$ is infeasible (otherwise, the result immediately follows). Choose any feasible frame schedule $C^{\star}$. Then, at frame $t_{i+1}<\infty$, we have the following twocases with positive probability: (a) from the finite improvement condition, $D\left(C\left[t_{i+1}\right], C^{\star}\right)<D\left(C\left[t_{i}\right], C^{\star}\right)$, or (b) $C\left[t_{i+1}\right]$ is feasible (i.e., $\left.D\left(C\left[t_{i+1}\right]=C^{\star \star}\right), C^{\star \star} \neq C^{\star}\right)$. First, if (b) is 


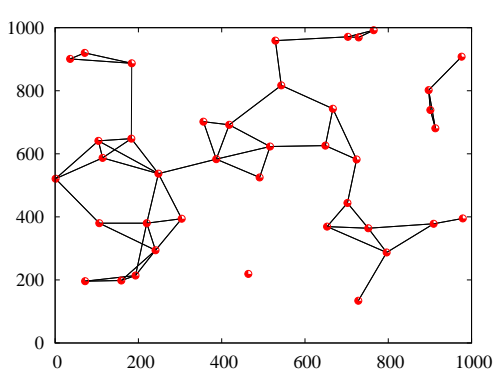

(a) Network topology and connectivity

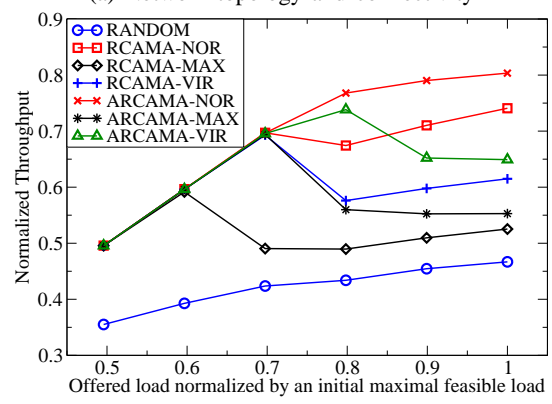

(c) Throughput for different loads

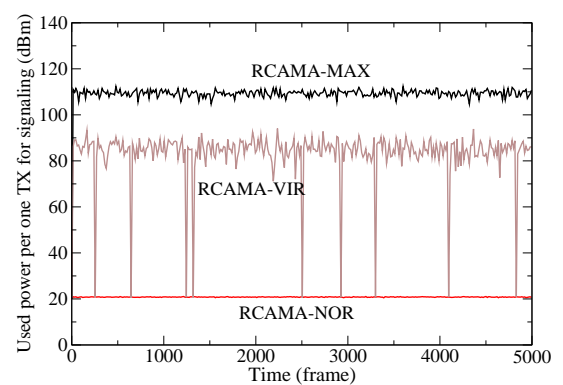

(b) Trace for energy usage for signaling

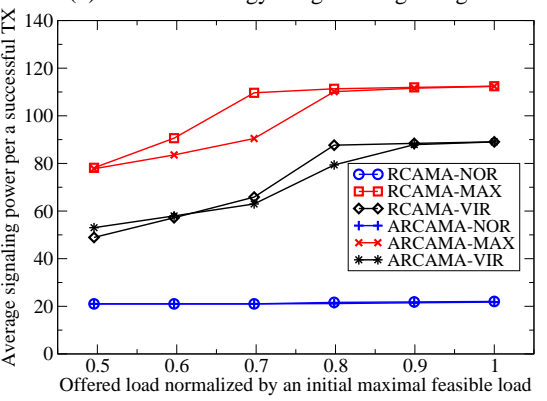

(d) Average energy per one successful TX

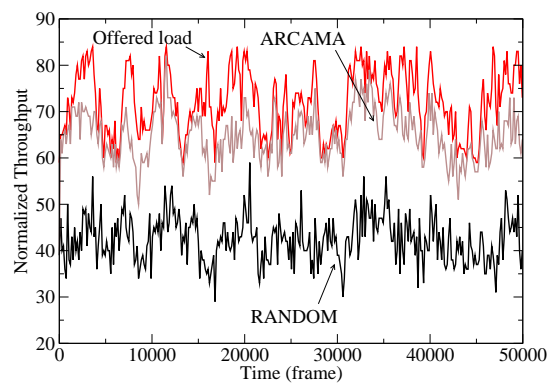

(a) Throughput traces: MLCT $=25$ frames

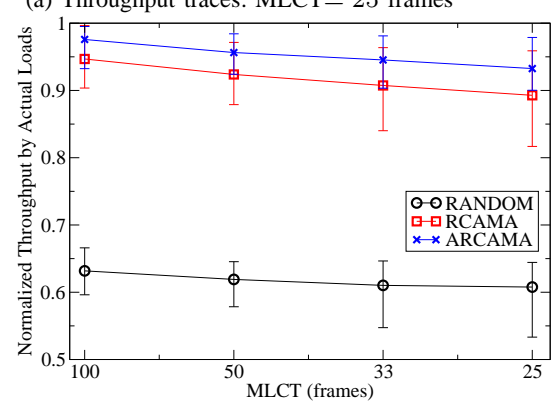

(b) Normalized throughput for different MLCTs

Fig. 6. Steady-state throughput and energy usage

Fig. 7. Adaptation to load changes

true, the result immediately follows. Second, if (a) is true, we are also done, since $D\left(C[0], C^{\star}\right)$ is upper-bounded by $\sum_{l=1}^{|\mathcal{L}|} \theta_{l}$. This completes the proof.

Proof of Theorem III.1(ii): Let $n_{\theta}=\sum_{l=1}^{|\mathcal{L}|} \theta_{l}$ be the total number of loads. We first note that the total number of frame schedules and $n_{\theta}$ are fixed, the number of frames to decrease the distance from a certain infeasible schedule to a feasible schedule (i.e., $t$ in Definition III.4) is uniformly bounded by a finite number of frames, and depends only on the scheduling algorithm, which we denote by $T_{\Pi}$. For simplicity, we use $T$ to refer to $T_{\Pi}$ here in this proof.

Recall that the proof of Theorem III.1(i) implies that for the current frame $i$, we have

$$
\operatorname{Pr}\left\{D\left(C[i+T], C^{\star}\right)=D\left(C[i], C^{\star}\right)-1\right\} \geq(1 / F)^{T n_{\theta}},
$$

i.e., over an interval of two frames, with at least probability $q=(1 / F)^{T n_{\theta}}, D\left(C[i], C^{\star}\right)$ decreases by 1 , since in the worst cast, each transmission was unsuccessful, and a time-slot is randomly chosen for each transmission with probability $1 / F$, and we nave $n_{\theta}$ number of scheduled transmissions, and this happens over two frames.

This also implies that with at least probability of $q^{D\left(C[0], C^{\star}\right)}$, the system will converge to $C^{\star}$ within $T \times$ $D\left(C[0], C^{\star}\right)$ frames. Note that convergence to a feasible schedule (possibly different from $C^{\star}$ ) could occur much earlier, since there could be multiple feasible frame schedules.

Further, note that for any frame schedule $C$, we have

$$
D\left(C, C^{\star}\right) \geq n_{\theta} .
$$

Thus, we have for any frame schedule $C$, we have $\forall C \in$ $\mathcal{C}, \forall C^{\star} \in \mathcal{C}^{\star}$ (where $\mathcal{C}$ and $\mathcal{C}^{\star}$ is the set of all possible and feasible frame schedules, respectively),

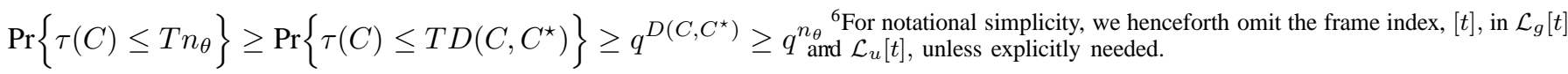

Now, let us study the evolution of the frame schedule at frames $\left\{0, T n_{\theta}, 2 T n_{\theta}, \cdots\right\}$. Observe that at any frame $T t \cdot n_{\theta}$ it will converge to a feasible frame schedule within up to frame $T(t+1) \cdot T n_{\theta}$ with probability at least $q^{n_{\theta}^{2}}$. This immediately provides a exponential upper bound on the convergence rate, i.e., $\forall C \in \mathcal{C}$,

$$
\operatorname{Pr}\left\{\tau(C)>t T n_{\theta}\right\} \leq\left(1-q^{n_{\theta}^{2}}\right)^{t} .
$$

By letting $p=1-q^{n_{\theta}^{2}}$, and $K=T n_{\theta}$, the result follows.

\section{Proof of Theorem V.1}

Proof of Theorem V.1(i): First, we will prove the finite sustenance condition. Note that from Rule IV.1, the time-slots for the scheduled TXs that were successful are sustained in the same position at the next frame. Clearly, if all the scheduled TXs were successful (i.e., C $[t]$ reaches a feasible FS), then the frame schedule at the current frame would be same as that at the previous frame. Thus, the finite sustenance condition is satisfied. In what follows, we focus on the proof of the finite improvement condition.

Let the current frame be $t$, and choose an arbitrary feasible FS $C^{\star}$. Since $C[t]$ is not feasible (otherwise the result immediately follows), there exists some link $l$, such that the TX over $l$ on some time-slot $s$ is not successful at this frame $t$. Let us denote the set of such "unsatisfied links" by $\mathcal{L}_{u}[t]$. Also, denote by $\mathcal{L}_{g}[t]$ the set of links with "good" position w.r.t. $C^{\star}$, i.e., the set of links whose slot schedules (i.e., row vector of a FS) are equal to those in $C^{\star} 6$. We first choose such an $l \in \mathcal{L}_{u} \backslash \mathcal{L}_{g}$ if $\mathcal{L}_{u} \backslash \mathcal{L}_{g} \neq \emptyset$, and choose $l \in \mathcal{L}_{g}$ otherwise.

Case 1: $l \in \mathcal{L}_{u} \backslash \mathcal{L}_{g}$. In this case, we again consider two sub-cases based on whether $c_{l s}^{\star}=0$ or 1 (i.e., whether the 
unsuccessful TX over $l$ on time-slot $s$ is scheduled $C^{\star}$ or not).

(i) $c_{l s}^{\star}=0$. Since $l \notin \mathcal{L}_{g}$, there is a slot $s^{\prime} \neq s$, such that $c_{l s^{\prime}}^{\star}=1$ and $c_{l s^{\prime}}[t]=0$. Note that the slot schedules of $l$ in $C[t]$ and $C^{\star}$ must have the same number of 1 's. Now from Property VII.1, there is a positive probability that at frame $t+1$ we have $C[t+1]$, such that the scheduled transmission over $l$ on slot $s$ at frame $t$ is moved to slot $s^{\prime}$, and all other scheduled transmissions at frame $t+1$ are scheduled at the same slots as at frame $t$. Then, we have $D\left(C[t+1], C^{\star}\right)=D\left(C[t], C^{\star}\right)-1$.

(ii) $c_{l s}^{\star}=1$. We first let $\mathcal{L}_{s}^{\prime}$ denote the set of scheduled links on $s$ by $C[t]$, but not by $C^{\star}$, i.e., $\mathcal{L}_{s}^{\prime}=\left\{i \in \mathcal{L} \mid c_{i s}=\right.$ $\left.1, c_{i s}^{\star}=0\right\}$.

Then, again there are two sub-cases: (a) there exists a unsuccessful link $l^{\prime} \in \mathcal{L}_{s}^{\prime}, l^{\prime} \neq l$, or (b) all the scheduled links in $\mathcal{L}_{s}^{\prime}$ are successful on $s$.

(a): Similar to (i), we can move the unsuccessful TX over $l^{\prime}$ on $s$ to a time-slot $s^{\prime} \neq s$, on which a TX is scheduled by $\left.C^{\star}\right)$, since $\mathcal{L}_{s}^{\prime} \cap \mathcal{L}_{g}=\emptyset$. Then, we have $D\left(C[t+1], C^{\star}\right)=D\left(C[t], C^{\star}\right)-1$.

(b): In this sub-case, we first have the following claim (the proof will be presented later):

Claim VII.1. Suppose that we choose a FS at frame $t$, such that $C[t+1]=C[t]$ (which is possible from Property VII.1), i.e., $D\left(C[t+1], C^{\star}\right)=D\left(C[t], C^{\star}\right)$. Then, there exists a link $l^{\prime} \in \mathcal{L}_{s}^{\prime}[t+1]$, such that the scheduled TX over $l^{\prime}$ on secomes unsuccessful.at frame $t+1$.

If Claim VII.1 is true, then, at the frame $t+1, l^{\prime}$ corresponds to Case 1(i). Thus, after two frames from $t$, we have $D\left(C[t+2], C^{\star}\right)=D\left(C[t], C^{\star}\right)-1$.

Case 2: $l \in \mathcal{L}_{g} \subset \mathcal{L}_{u}$. Note that the fact we are in this case implies that all the links in $\mathcal{L}_{u} \backslash \mathcal{L}_{g}$ are satisfied, since we always choose first an unsatisfied link in $\mathcal{L}_{u} \backslash \mathcal{L}_{g}$ by construction.

Then, using the same definition of $\mathcal{L}_{s}^{\prime}$ as that in Case 1(ii), this case corresponds to Case 1(ii)(b), i.e., when all the links in $\mathcal{L}_{s}^{\prime}$ are successful on slot $s$. Thus, again based on Claim VII.1, with a positive probability we have $D\left(C[t+2], C^{\star}\right)=$ $D\left(C[t], C^{\star}\right)-1$. This completes the proof. Now, it remains to prove Claim VII.1.

Proof of Claim VII.1: By hypothesis (i.e., Case 1(ii)(b)), all the scheduled links on $s$ are successful except for $l$. In other words, all the links in $\mathcal{L}_{g} \cup \mathcal{L}_{s}^{\prime}[t]$ are successful except for $l$. Note that $c_{l s}^{\star}=1$ and $l \in \mathcal{L}_{u}$. This implies that the aggregate interference by TXs over the links in $\mathcal{L}_{g}$ that are scheduled on $s$ is not enough to make the TX over l on $s$ unsuccessful, and the TXs in $\mathcal{L}_{s}^{\prime}[t] \backslash\{l\}$ necessarily contribute to the TX failure over $l$.

Also, since $C[t+1]=C[t]$ by assumption, all the scheduled links on $s$ except for $l$ should have low priority at frame $t+1$. Then, HPC condition implies that the high priority valid TX at stage 1 (in fact, the TX over $l$ should be valid at stage 1) is guaranteed to be successful, there must an unsuccessful TX over a link in $\mathcal{L}_{s}^{\prime}[t]$ at frame $t+1$.
Proof of Theorem V.1(ii): It is clear that with any $\left(\vec{Q}_{s}^{A}[t]\right)$, where $\vec{Q}_{s}^{A}[t] \geq \vec{P}_{s}^{A}[t], s=1, \ldots, F, t=0,1, \ldots$, in elementwise, more low priority TXs will be invalidated at stage 3 . Note that irrespective of the signaling power adjustment scheme, the set of high priority TXs which have data transmission is fixed. Thus, RCAMA satisfies HPC with $\left(\vec{Q}_{s}^{A}[t]\right)$. This completes the proof.

\section{Proof of Theorem V.2}

To prove that RCAMA-VIR satisfies HPC subject to the condition on $d_{m i n}$, it suffices to show that for arbitrary high priority TX in $\mathcal{H}_{F}^{2}$, its success is guaranteed. In this proof, we consider the case of RTS-decoding failure of a high priority TX in $\mathcal{H}_{F}^{2}$. Similar proof can be applied to the case of CTSdecoding failure.

We consider a high priority TX over over the link from $A$ to $B$, and a set of most dominant $N_{\text {int }}$ low priority TXs over $C_{i} \rightarrow D_{i}, i=1, \ldots, N_{i n t}$. Note that we have more high and low priority TXs in the network. Suppose that RTS from $A$ to $B$ fails due to the aggregate interference by RTSs from $C_{i}, i=1, \ldots, N_{i n t}$. (see Figure 5 for a similar scenario with only differences that more high and low priority TXs are in the network and $N$ is replaced by $N_{\text {int }}$ ).

First, from (4) we have that

$$
\hat{I}_{d(l)}^{r} \leq I_{d(l)}^{r} .
$$

Note that the exact aggregate interference by RTS messages from $C_{1}, \ldots, C_{N_{i n t}}$ is given by:

$$
I_{r}^{l}=P \times \sum_{i=1}^{N_{i n t}} \frac{1}{\left(y_{i}\right)^{\alpha_{i}}}
$$

where $\alpha_{i}$ is the path loss exponent from $C_{i}$ to $B$. Then, from (5) and (6), we have

$$
\hat{I}_{d(l)}^{r} \leq I_{d(l)}^{r} \leq P \times \sum_{i=1}^{N_{i n t}} \frac{1}{\left(y_{i}\right)^{\underline{\alpha}}} .
$$

Now, let $y^{\prime}=d\left(B, C^{\prime}\right)$, which is computed by $B$ as follows:

$$
\left(y^{\prime}\right)^{\alpha_{v}}=\frac{P}{\hat{I}_{d(l)}^{r}} \geq 1 / \sum_{i=1}^{N_{\text {int }}} \frac{1}{\left(y_{i}\right) \underline{\alpha}},
$$

where $\alpha_{v}$ is the path loss exponent from $B$ to $C^{\prime}$.

As described in the algorithm description, $B$ assumes that there is no signal power loss between $C^{\prime}$ and $D^{\prime}$. Based on such assumption and $y^{\prime}, B$ will adjust its CTS message power (denoted by $P_{c}^{v}$ ) enough to invalidate the CTS from $D^{\prime}$ to $C^{\prime}$. From (8), this is given by:

$\frac{P}{P_{c}^{v} /\left(y^{\prime}\right)^{\alpha_{v}}} \leq \gamma \Rightarrow P_{c}^{v} \geq \frac{P\left(y^{\prime}\right)^{\alpha_{v}}}{\gamma} \geq \frac{P}{\gamma \sum_{i=1}^{N_{i n t}} 1 /\left(y_{i}\right) \underline{\alpha}}$

Let the path loss exponent from $D_{i}$ to $C_{i}$ be $\beta_{i}$. Then, the SINR value at $C^{\prime}$ for its CTS message from $D^{\prime}$ will be:

$$
\frac{P /\left(z_{i}\right)^{\beta_{i}}}{Z_{H}+P_{c}^{v} /\left(y_{i}\right)^{\alpha_{i}}}
$$

where $Z_{H}$ is the total received power at $C^{\prime}$ by other high and low priority TXs except for TXs over $A \rightarrow B$ and $C_{i} \rightarrow D_{i}, i=$ $1, \ldots, N_{\text {int }}$. 
Then, it suffices to show that with $P_{c}^{v}$, all of $N_{\text {int }}$ low priority TXs over $C_{i} \rightarrow D_{i}, i=1, \ldots, N_{i n t}$ are invalidated, i,e.,

$$
\frac{P /\left(z_{i}\right)^{\beta_{i}}}{Z_{H}+P_{c}^{v} /\left(y_{i}\right)^{\alpha_{i}}} \leq \frac{P /\left(z_{i}\right)^{\beta_{i}}}{P_{c}^{v} /\left(y_{i}\right)^{\alpha_{i}}}=\frac{P\left(y_{i}\right)^{\alpha_{i}}}{P_{c}^{v}\left(z_{i}\right)^{\beta_{i}}} \leq \gamma .
$$

Now, we have

$$
\begin{aligned}
\frac{P /\left(z_{i}\right)^{\beta_{i}}}{Z_{H}+P_{c}^{v} /\left(y_{i}\right)^{\alpha_{i}}} & \leq \frac{P\left(y_{i}\right)^{\alpha_{i}}}{P_{c}^{v}\left(z_{i}\right)^{\beta_{i}}} \leq \frac{P\left(d_{i n t}\right)^{\alpha_{i}}}{P_{c}^{v}\left(d_{\text {min }}\right)^{\beta_{i}}} \leq \frac{P\left(d_{i n t}\right)^{\bar{\alpha}}}{P_{c}^{v}\left(d_{\text {min }}\right)^{\underline{\alpha}}} \\
& \leq \frac{\gamma\left(d_{i n t}\right)^{\bar{\alpha}} \sum_{i=1}^{N_{i n t}} 1 /\left(y_{i}\right)^{\underline{\alpha}}}{\left(d_{\text {min }}\right)^{\underline{\alpha}}} \quad \text { (from (9)) } \\
& \leq \gamma \frac{N_{\text {int }}\left(d_{i n t}\right)^{\bar{\alpha}}}{\left(d_{\text {min }}\right)^{2} \underline{\alpha}} .
\end{aligned}
$$

Thus, if $\sqrt[2 \alpha]{N_{i n t}}\left(d_{i n t}\right)^{\bar{\alpha} /(2 \underline{\alpha})} \leq d_{\text {min }}$, we have

$$
\frac{N_{i n t}\left(d_{i n t}\right)^{\bar{\alpha}}}{\left(d_{\text {min }}\right)^{2} \underline{\alpha}} \leq 1 \text {. }
$$

Thus, (10) is proved, which completes the proof.

\section{REFERENCES}

[1] Y. Yi, G. de Veciana, and S. Shakkottai, "On optimal MAC scheduling with physical interference," in Proceedings of IEEE Infocom, Anchorage, AK, 2007.

[2] L. Tassiulas and A. Ephremides, "Stability properties of constrained queueing systems and scheduling for maximum throughput in multihop radio networks," IEEE Transactions on Automatic Control, vol. 37, no. 12, pp. 1936-1949, December 1992.

[3] P. Chaporkar, K. Kar, and S. Sarkar, "Throughput guarantees through maximal scheduling in wireless networks," in Proceedings of the 43rd Annual Allerton Conference on Communication, Control and Computing, 2005.

[4] X. Lin and N. B. Shroff, "The impact of imperfect scheduling on crosslayer rate control in wireless networks," in Proceedings of INFOCOM, 2005.

[5] X. Wu and R. Srikant, "Bounds on the capacity region of multi-hop wireless networks under distributed greedy scheduling," in Proceedings of INFOCOM, 2006.

[6] G. Sharma, N. B. Shroff, and R. R. Mazumdar, "Joint congestion control and distributed scheduling for throughput guarantees in wireless networks," in Proceedings of IEEE Infocom, 2007.

[7] G. Sharma, R. R. Mazumdar, and N. B. Shroff, "On the complexity of scheduling in wireless networks," in Proceedings of MOBICOM, 2006.

[8] E. Modiano, D. Shah, and G. Zussman, "Maximizing throughput in wireless networks via gossiping," in Proceedings of ACM Sigmetrics, New York, NY, USA, 2006.

[9] A. Eryilmaz, A. Ozdaglar, and E. Modiano, "Polynomial complexity algorithms for full utilization of multi-hop wireless networks," in Proceedings of IEEE Infocom, 2007.

[10] X. Lin and S. Rasool, "Constant-time distributed scheduling policies for ad hoc wireless networks," in Proceedings of IEEE Conference on Decision and Control, 2006.

[11] C. Joo and N. B. Shroff, "Performance of random access scheduling schemes in multi-hop wireless networks," in Proceedings of IEEE Infocom, 2007.

[12] P. While, "RSVP and integrated services in the internet: A tutorial," IEEE Communications Magazine, May 1997.

[13] Y. Yi, G. de Veciana, and S. Shakkottai, "Mac scheduling with low overheads by learning neighborhood's contention patterns," Princeton University, Tech. Rep., 2007.

[14] Y. S. P. Gupta and A. Stolyar, "Random-access scheduling with service differentiation in wireless networks," in Proceeding of INFOCOM, March 2005.

[15] L. Tassiulas, "Linear complexity algorithms for maximum throughput in radionetworks and input queued switches," in Proceedings of IEEE INFOCOM, 1998.

[16] S. Sanghavi, L. Bui, and R. Srikant, "Distributed link scheduling with constant overhead," in Proceedings of ACM Sigmetrics, 2007.

[17] Y. Yi, A. Proutiere, and M. Chiang, "Complexity-stability-delay tradeoff in scheduling over wireless network," Preprint. Available at http://www.princeton.edu/ỹyi/tradeoff.pdf, 2007.
[18] K. Jung and D. Shah, "Low delay scheduling in wireless network," in Proceeding of ISIT, 2007.

[19] A. Behzad and I. Rubin, "Optimum integrated link scheduling and power control for ad hoc wireless networks," IEEE Transactions on Vehicular Technology, 2006, to appear.

[20] R. Negi and A. Rajeswaran, "Physical layer effect on mac performance in ad-hoc wireless networks," in In Proceedings of Communications, Internet, and Information Technology, 2003.

[21] P. Björklund, P. Värbrand, and D. Yuan, "Resource optimization of spatial TDMA in ad hoc radio networks: A column generation approach." in Proceedings of IEEE INFOCOM, 2003.

[22] T. Moscibroda and R. Wattenhofer, "The complexity of connectivity in wireless networks," in Proceedings of INFOCOM, 2006.

[23] R. Cruz and A. Santhanam, "Optimal routing, link scheduling and power control in multi-hop wireless networks," in Proceeding of INFOCOM, 2003.

[24] K. Jain, J. Padhye, V. N. Padmanabhan, and L. Qiu, "Impact of interference on multi-hop wireless network performance," in Proceedings of ACM MOBICOM, 2003.

[25] I. Rhee, A. Warrier, M. Aia, and J. Min, "Z-mac: a hybrid mac for wireless sensor networks," in Proceedings of the ACM conference on Embedded networked sensor systems (SenSys), 2005.

[26] M. Krunz, A. Muqattash, and S. Lee, "Transmission power control in wireless ad hoc networks: Challenges, solutions, and open issues," IEEE Network, vol. 18, no. 5, pp. 8-14, 2004.

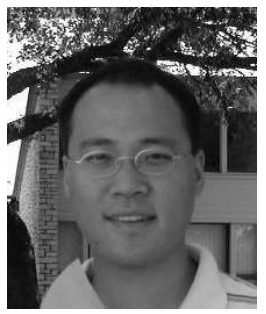

Yung Yi Yung Yi received his B.S. and M.S. in the School of Computer Science and Engineering from Seoul National University, South Korea in 1997 and 1999, respectively, and his Ph.D. in the Department of Electrical and Computer Engineering at the University of Texas at Austin. Now he is a post-doctoral research associate in the Department of Electrical Engineering at Princeton University. His current research interests include scheduling and QoS for wireless networks, congestion control in the Internet, and design and performance analysis of computer networks. His email address is yyi@princeton.edu.

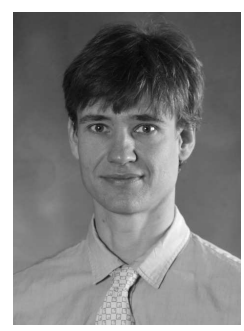

Gustavo de Veciana Gustavo de Veciana (S'88M'94-SM 2001) received his B.S., M.S, and Ph.D. in electrical engineering from the University of California at Berkeley in 1987, 1990, and 1993 respectively. He is currently a Professor at the Department of Electrical and Computer Engineering at the University of Texas at Austin and the Director of the Wireless Networking and Communications Group (WNCG). His research focuses on the design, analysis and control of telecommunication networks. Current interests include: measurement, modeling and performance evaluation; wireless and sensor networks; architectures and algorithms to design reliable computing and network systems. Dr. de Veciana has been an editor for the IEEE/ACM Transactions on Networking. He is the recipient of General Motors Foundation Centennial Fellowship in Electrical Engineering and a 1996 National Science Foundation CAREER Award, co-recipient of the IEEE William McCalla Best ICCAD Paper Award for 2000, and co-recipient of the Best Paper in ACM Transactions on Design Automation of Electronic Systems, Jan 2002-2004.

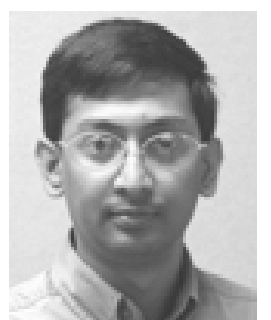

Sanjay Shakkottai Sanjay Shakkottai (M'02) received his Ph.D. from the University of Illinois at Urbana-Champaign in 2002. He is currently with The University of Texas at Austin, where he is an Associate Professor in the Department of Electrical and Computer Engineering. He was the finance chair of the 2002 IEEE Computer Communications Workshop in Santa Fe, NM. He received the NSF CAREER award in 2004. His research interests include wireless and sensor networks, stochastic processes and queueing theory. His email address isshakkott@ece.utexas.edu. 